\title{
Expression and prognostic value of E2F3 transcription factor in non-small cell lung cancer
}

\author{
LEI WU ${ }^{1 *}$, SHAN WAN $^{1 *},{\text { JINFAN } L^{2,3 *}, \text { YIYING XU }}^{1}$, XIAOLI LOU ${ }^{1}$, \\ MAOMIN SUN $^{4 * *}$ and SHOULI WANG ${ }^{1,3^{* *}}$ \\ ${ }^{1}$ Department of Pathology, School of Biology and Basic Medical Sciences, Soochow University, Suzhou, \\ Jiangsu 215123; ${ }^{2}$ Department of Pathology, The Second Affiliated Hospital of Zhejiang University School of Medicine, \\ Hangzhou, Zhejiang 310009; ${ }^{3}$ Department of Collaborative Innovation Center of Clinical Immunology \\ between Soochow University and Sihong People's Hospital, Sihong, Jiangsu 223900; ${ }^{4}$ Laboratory Animal Research Center, \\ Soochow University School of Medicine, Suzhou, Jiangsu 215123, P.R. China
}

Received May 5, 2020; Accepted January 20, 2021

DOI: $10.3892 / \mathrm{ol} .2021 .12672$

\begin{abstract}
E2F transcription factor 3 (E2F3) plays a vital role in the development of various types of cancer. To verify whether E2F3 is a suitable biomarker for the prognosis of lung cancer, bioinformatics analysis was performed to determine the differential expression level of E2F3 in lung cancer and the surrounding non-tumor tissues, and the results were confirmed in a NSCLC cell line and a tissue microarray (TMA). The relevance of E2F3 in non-small cell lung cancer (NSCLC) was investigated in 19 studies from the Oncomine database and confirmed in The Cancer Genome Atlas database. In the lung cancer cell line A549, the inhibition of E2F3 mRNA expression level led to decreased tumor cell viability and cell migration, which was determined by a Cell Counting Kit-8 and wound healing assays, respectively. Immunohistochemistry analyses of E2F3, Bcl-2, Bax and caspase- 3 were performed in the NSCLC TMA $(n=50)$. The assessment of TMA detected the increase of E2F3 protein expression level in the tumor tissues, as compared with that in the non-tumor tissues, which was also correlated with the increase in expression of $\mathrm{Bcl}-2$ in tumors. Analysis of the clinical data from patients with NSCLC revealed that the overexpression of E2F3 was associated with early lymphatic
\end{abstract}

Correspondence to: Professor Shouli Wang, Department of Pathology, School of Biology and Basic Medical Sciences, Soochow University, 199 Ren'ai Road, Suzhou, Jiangsu 215123, P.R. China E-mail: wangsoly112@hotmail.com

Professor Maomin Sun, Laboratory Animal Research Center, Soochow University School of Medicine, Suzhou, Jiangsu 215123, P.R. China

E-mail: sunmaomin@suda.edu.cn

*****Contributed equally

Key words: lung cancer, E2F3, bioinformatics, survival rate, metastasis, prognosis spreading, and poor patient survival time. The OncomiR website was used to predict the E2F3 upstream microRNAs and determine their prognostic value in patients with NSCLC. The results from the present study revealed that E2F3 was overexpressed at both the transcriptional and translational levels in NSCLC tissues, as compared with that in non-tumor tissues. The overexpression of E2F3 was associated with the upregulation of the anti-apoptotic factor, Bcl-2, which may contribute to uncontrolled tumor growth. Thus, E2F3 was shown to have important oncogenic properties in the development of NSCLC, and it may become a potential biomarker for patients with NSCLC.

\section{Introduction}

The latest epidemiological data in 2018 showed that lung cancer had the highest incidence rate among all malignancies in China, with $\sim 781,000$ cases recorded annually (1). Lung cancer includes a variety of tumor entities, with non-small cell lung cancer (NSCLC) being the most common. More specifically, the histological types of NSCLC include adenocarcinoma, squamous cell carcinoma and large cell carcinoma, which have distinct pathological and clinical features (2). The 5-year survival time of patients with NSCLC at stage IIIB/IV was only $\sim 15 \%$ in the United States in 2010, revealing the urgent demand for a new and improved therapeutic approach, as well as biomarkers for prognosis prediction (2). During the last decade, progress has been made in the treatment of adenocarcinoma following the discovery of epidermal growth factor receptor (EGFR) (3). Tyrosine kinase inhibitors (TKIs), targeting EGFR and its downstream signaling proteins, have changed conventional chemotherapy and radiotherapy towards the development of targeted therapy in lung cancer (3). Except for alterations in EGFR, KRAS oncogene mutations and MET gene amplification have also been shown to play an important role in the progression of lung cancer, and have contributed to the development of biomarker and targeted therapy (4). Despite the improvements in targeted therapy for lung cancer, certain subgroups of patients with lung cancer show little response to targeted therapy, leading to a 5-year survival rate 
of $<20 \%$ (3). Therefore, a comprehensive understanding of molecular targets and the development of new biomarkers are necessary for the improvement of therapeutic approaches for NSCLC.

Bioinformatics is an efficient and convenient approach, that connects certain genes with the biological behavior and clinical outcomes of different tumors (5). For example, Oncomine is a tumor gene chip-based database, that integrates RNA and DNA-sequencing data from multiple databases and published literature, covering 65 gene chip datasets, 4,700 chips and 480 million gene expression data (6). Oncomine can also perform co-expression analysis to show which genes in the dataset are similarly expressed to the selected target gene and provide correlation values. It can identify an oncogene expression profile, where high gene expression is found in a fraction of samples in the total population within a cancer type, using Cancer Outlier Profile Analysis. This database can also be used to analyze the mRNA expression level of target genes in different tumors and enable comparisons with non-tumor tissues. Furthermore, the setup of the analysis can be specified to important clinical annotations, including secreted proteins, kinases, membrane proteins and known molecular drug targets, which facilitate the discovery of new biomarkers and therapeutic targets (6). Oncomine also provides access to important clinical parameters, such as tumor size, tumor cell differentiation, metastasis and survival time (6). In addition to the Oncomine database, the Kaplan-Meier survival analysis database integrates gene expression data with clinical information, in particular survival data (7). The tumor types included in this database mainly include lung, breast, gastric and cervical cancer (7). The database also provides an appropriate grouping of patients, according to the expression level of a specific gene, which enables the association of the specific gene with patient survival time (7). MicroRNAs (miRNAs/miRs) are small RNAs, that play an important role in tumorigenesis, development and survival $(8,9)$. The OncomiR website is an online resource that contains information on miRNA research associated with $>30$ different cancer types, and provides tools for the identification and validation of miRNAs without the requirement for a professional computer language foundation (10). The tumor database contains miRNA-Sequencing (Seq) and RNA-Seq information and clinical data from nearly 10,000 patients with cancer, and enables connections with tumor development, staging and overall survival (OS) time (10). Bioinformatics analysis based on the online databases described enables the identification of potential molecular targets in NSCLC tumorigenesis.

$\mathrm{E} 2 \mathrm{~F}$ transcription factor $(\mathrm{E} 2 \mathrm{~F})$ proteins are defined as a family of transcription regulators, that are involved in numerous cellular processes, such as cell cycle progression, DNA replication, DNA repair and apoptosis (11). Different E2F family members have various effects on the initiation and progression of several types of cancer. Typically, the E2F family members are divided into transcriptional activators (E2F1-E2F3) or transcriptional repressors (E2F4-E2F8) of target genes (12). E2F3, a positive regulator, has been shown to interact with histone acetyltransferase, and be involved in DNA replication by stimulating the entry of quiescent cells into the $\mathrm{G}_{1} / \mathrm{S}$ phase of the cell cycle $(13,14)$. Specifically, E2F3 mRNA encodes two isoforms, E2F3a and E2F3b, both of which contribute to the activation of E2F3 target gene transcription; however, the 2 isoforms of E2F3 showed different activity in regulating biological functions, such as cell proliferation $(14,15)$. A previous study found that the downregulation of E2F3 using short-hairpin RNA (shRNA) could suppress cell proliferation, migration and invasion ability of the DU145 prostate cancer cell line (14). However, another previous study has focused on the impact of $\mathrm{E} 2 \mathrm{~F} 3$ on the tumorigenesis and prognosis in patients with NSCLC (13).

The present study aimed to investigate if E2F3 was differentially expressed in the NSCLC tissues compared with non-tumor lung tissues by using a bioinformatics approach. By analyzing the level of E2F3 protein in lung cancer cell line and patient tissues, the importance and potential value of E2F3 were assessed in patients with NSCLC as a potential biomarker. Functional analysis was used to analyze the effect of E2F3 on tumor cell proliferation and apoptosis to ascertain the underlying mechanism of how E2F3 was involved in the tumorigenesis of NSCLC. The present study revealed a new aspect of E2F3 in promoting NSCLC progression, which emphasized the value of E2F3 as a potential biomarker for the prediction of prognosis in patients with NSCLC.

\section{Materials and methods}

Patient material. A total of 50 pairs of lung cancer tissues and adjacent normal lung tissues, which were $>2 \mathrm{~cm}$ away from the tumor tissues, were collected from patients with lung cancer, between January, 2016 and January, 2017 at the Department of Pathology of The Second Affiliated Hospital of Soochow University (Jiangsu, China). All patients underwent standard lung resection plus lymphadenectomy by the same surgical team, and no patients received any preoperative therapy. All sample collection processes were performed with the permission of the ethical review committee of Soochow University, and standard operating procedures were strictly followed for the processing of tissue samples. The collected tissues were immediately stored at $-80^{\circ} \mathrm{C}$ until further use. The clinicopathological features of the patients are provided in Table I. Histological assessment of tumor differentiation and clinical stages were confirmed by the World Health Organization guidelines previously described in lung cancer studies (3). Histological classification of collected lung cancer tissue include squamous cell lung carcinoma (LUSC, $n=23$ ) and lung adenocarcinoma (LUAD, n=27). The collection of tissue samples from patients with lung cancer, for the present study, was approved by the Ethics Committee of Soochow University (Jiangsu, China). All patients involved were informed before the collection of tissue samples. Written informed consent was obtained from all patients for their participation in the study, in accordance with the 1964 Declaration of Helsinki and its later amendments.

Tissue microarray (TMA) preparation. Tissue samples were fixed in $10 \%$ formalin for 2 days at room temperature. Paraffin embedded tissues were cut in 2-3 $\mu \mathrm{m}$ sections using a microtome and dried overnight. The tissue slides were routinely stained with eosin $(30 \mathrm{sec})$ and hematoxylin ( $2 \mathrm{~min}$ ) at room temperature for the confirmation of the diagnosis of each sample by pathologists under a light microscope (magnifications, x100 
Table I. Association between E2F3 and clinicopathological parameters in patients with lung cancer.

E2F3 expression level

\begin{tabular}{|c|c|c|c|c|}
\hline \multirow[b]{2}{*}{ Variables } & \multirow[b]{2}{*}{ Number } & & \multirow[b]{2}{*}{ P-value } \\
\hline & & High n=29 & Low n=21 & \\
\hline Sex & & & & 0.815 \\
\hline Male & 30 & $17(56.7 \%)$ & $13(43.3 \%)$ & \\
\hline Female & 20 & $12(60.0 \%)$ & $8(40.0 \%)$ & \\
\hline Age, years & & & & 0.261 \\
\hline$\leq 60$ & 17 & $8(47.1 \%)$ & $9(52.9 \%)$ & \\
\hline$>60$ & 33 & $21(63.6 \%)$ & $12(36.4 \%)$ & \\
\hline Tumor size, $\mathrm{cm}$ & & & & 0.083 \\
\hline$\leq 2$ & 3 & $2(66.7 \%)$ & $1(33.3 \%)$ & \\
\hline $2-5$ & 27 & $8(29.6 \%)$ & $19(70.4 \%)$ & \\
\hline$>5$ & 20 & $12(60.0 \%)$ & $8(40.0 \%)$ & \\
\hline Lymphatic metastasis & & & & 0.003 \\
\hline Yes & 29 & $22(75.9 \%)$ & $7(24.1 \%)$ & \\
\hline No & 21 & $7(33.3 \%)$ & $14(66.7 \%)$ & \\
\hline TNM stage & & & & 0.979 \\
\hline I-II & 38 & $22(57.9 \%)$ & $16(42.1 \%)$ & \\
\hline III-IV & 12 & $7(58.3 \%)$ & $5(41.7 \%)$ & \\
\hline
\end{tabular}

and $\mathrm{x} 400$ ). The tumor area and surrounding non-tumor tissues in paraffin were drilled out and transferred to an acceptor of a predesigned array using a puncher. After all tissue samples from the original paraffin blocks were transferred to the microarray acceptor, the complete tissue microarray was briefly warmed at $65^{\circ} \mathrm{C}$ in an oven for $30 \mathrm{~min}$. When slightly dissolved by heating, the tissue microarray was reformed in a plastic cassette. Each dot on the microarray had a diameter of $2 \mu \mathrm{m}$, which was $1 \mu \mathrm{m}$ away from adjacent dots.

Immunohistochemistry (IHC). Tissue samples from patients were fixed with $10 \%$ formalin for 2 days at room temperature, followed by paraffin embedment for TMA preparation. The tissue sections (2-3 $\mu \mathrm{m})$ from TMA were deparaffinized and rehydrated using the following washing steps: $3 \times 5$ min xylene, $2 \times 3$ min $100 \%$ ethanol, $3 \mathrm{~min} 95 \%$ ethanol and $3 \mathrm{~min} 75 \%$ ethanol, followed by rinsing with water. Sodium citrate buffer (10 mM; Ph 6.0; cat. no. AR0024; Boster Biological Technology) was incubated with the TMA using a microwave, twice for $15 \mathrm{~min}$. After antigen retrieval, the tissue sections were incubated with 3\% peroxidase (Yonghua Chemical Co., Ltd.) at room temperature for $10 \mathrm{~min}$, blocked with 5\% (w/v) BSA (Guangzhou Saiguo Biotech Co., Ltd.) at room temperature for $20 \mathrm{~min}$ and incubated with E2F3 primary antibody (cat. no. ab50917; dilution, 1:200; Abcam) overnight at $4^{\circ} \mathrm{C}$. The tissue sections were then incubated with a biotinylated anti-rabbit secondary antibody (cat. no. BA1003; Boster Biological Technology) at room temperature for $2 \mathrm{~h}$. Streptavidin-horseradish peroxidase (cat. no. BA1088; Boster Biological Technology) and peroxidase substrate solution (cat. no. MAX-001; MXB Biotechnologies) were added to the tissue sections at room temperature for 5-30 min for signal development. IHC stains were analyzed at $\mathrm{x} 100$ and $\mathrm{x} 400$ magnifications under a light microscope.
IHC of TMA was scored blindly by 2 pathologists from the Department of Pathology, The Second Affiliated Hospital of Zhejiang University School of Medicine, (Hangzhou, China) according to the intensity and percentage of positive tumor cells. Pathological stages of lung cancer were confirmed by the 2 pathologists according to the WHO guidelines of the NSCLC (3). Intensity was scored as follows: 0, Negative; 1 , low nuclear stain; 2, medium nuclear stain; 3, strong nuclear stain. The percentage of positivity was defined as follows: 0 , Negative; $1,<1 \% ; 2,<9 \% ; 3,10-50 \% ; 4,>51 \%$. The final score was calculated by multiplying the qualitative and quantitative parameters. Median value of IHC scores were analyzed in lung cancer tissue or adjacent lung tissue. Difference of median value of IHC scores were used to indicate the differential expression of proteins between lung cancer and adjacent tissue.

Western blot analysis. The total protein lysate was extracted from fresh tissue or cell lines using RIPA buffer (cat. no. 5871, Cell Signaling Technology, Inc.). The total protein concentration was measured using a BCA protein quantitative kit (Beyotime Institute of Biotechnology) and denatured by heating at $95^{\circ} \mathrm{C}$ for $10 \mathrm{~min}$. The protein samples $(50 \mu \mathrm{g})$ were separated using 8-12\% SDS-PAGE, transferred to a PVDF membrane (EMD Millipore), blocked with $5 \%(\mathrm{w} / \mathrm{v})$ skimmed milk in TBST $(0.1 \%$ Tween 20 in TBS, v/v) at room temperature for $1 \mathrm{~h}$, and incubated with the primary antibodies in the same blocking buffer against E2F3 (1:1,000; cat. no. ab152126; Abcam), caspase-3 (1:200; cat. no. ab32150; Abcam), Bcl-2 (1:1,000; cat. no. 12789-1-AP; ProteinTech Group, Inc.) and Bax (1:1,000; cat. no. 50599-2-Ig; ProteinTech Group, Inc.) overnight at $4^{\circ} \mathrm{C}$. Detection of $\beta$-actin $(1: 1,000$; cat. no. sc-47778; Santa Cruz Biotechnology Inc.) served as a loading control. 
Following which, the PVDF membrane was washed with TBS (0.1\% Tween-20) three times and incubated with a specific secondary antibody (dilution, 1:3,000; Boster Biological Technology) for $2 \mathrm{~h}$ at room temperature. Immunoreactivity was visualized using an ECL (Pierce; Thermo Fisher Scientific Inc.) detection system.

Reverse transcription-quantitative $(R T-q) P C R$. For total RNA extraction from cultured A549 cells, the NucleoSpin RNA II kit (Macherey-Nagel GmbH \& Co. KG) was used according to the manufacturer's instructions. A total of $2 \mu \mathrm{g}$ mRNA was used for cDNA synthesis using RevertAid $\mathrm{H}$ Minus Reverse Transcriptase $(0.9 \mu \mathrm{l}$; Thermo Fisher Scientific Inc.) according to manufacturer's instructions. Real-time PCR reactions were set up using the ABsolute qPCR SYBR Green ROX Mix (Thermo Fisher Scientific Inc.) and E2F3 specific primers (forward sequence, 5'-GTATGATACGTCTCTTGG TCTGC-3', reverse sequence, 5'-CAAATCCAATACCCC ATCGGG-3'). Glyceraldehyde-3-phosphate dehydrogenase (GAPDH, forward sequence, 5'-CAAGGTCATCCATGACAA CTTTG-3', reverse sequence, 5'-GTCCACCACCCTGTTGCT GTAG-3') was used as the housekeeping gene. Thermocycling conditions contained 1 cycle of initial denaturation at $95^{\circ} \mathrm{C}$ for $15 \mathrm{~min}, 40$ cycles of denaturation $\left(95^{\circ} \mathrm{C}\right.$ for $\left.15 \mathrm{sec}\right)$, followed by combined annealing and extension $\left(60^{\circ} \mathrm{C}\right.$ for $\left.60 \mathrm{sec}\right)$, and 1 cycle of melting curve at $60-95^{\circ} \mathrm{C}$ with increase of temperature at $0.5^{\circ} \mathrm{C} / \mathrm{min}$. The standard curve method was used for quantification. Different dilutions of cDNA from A549 cells $(1: 12.5,1: 25,1: 50,1: 100,1: 200$, and 1:400) were used for generation of the standard curves for quantification. Data was quantified the $2^{-\Delta \Delta \mathrm{Cq}}$ method (16).

Bioinformatics analysis. Oncomine database (6) was used to analyze the expression level of the E2F3 gene in the selected tumor types, including gastric cancer, lung cancer, breast cancer, hematological tumor and sarcoma. To determine the biological function of E2F3 in cancer, patient clinical data from the datasets were analyzed and characterized as 'cancer' and 'normal'. 'Ranking of genes' was used to identify the differential expression of E2F3 in different tumor types. The overexpression of E2F3 was compared between 19 NSCLC studies using meta-analysis, based on the fold change of the median levels of E2F3, as compared with non-tumor lung tissues.

The Kaplan-Meier plotter website (7) was used to compare survival rates in patients with NSCLC $(n=141)$ grouped by the expression levels of E2F3 and E2F3-associated miRNAs. The endpoint was either 5 years or the death of the patient. Time of patients without reaching the endpoint was shown as the follow-up periods. To confirm the expression data of E2F3 in lung cancer, The Cancer Genome Atlas (TCGA) database was used for the analysis (https://cancergenome.nih.gov/).

Using TCGA visualization website FireBrowse (http://firebrowse.org), the E2F3 expression levels were compared between the tumor and non-tumor tissues from the lung and squamous cell epithelium from tongue. The 'filter' included using lung adenocarcinoma (LUAD) and lung squamous cell carcinoma (LUSC). The expression data of the genes of interest was displayed as 'tumor vs. normal', according to the analysis filter. For the identification of E2F3-associated
miRNAs in lung cancer, the top 10 miRNAs targeting E2F3 were selected by using the Exiqon database (https:/www. exiqon.com/miRSearch). The OncomiR website (10) was used to analyze the expression level of the top 10 miRNAs in LUAD and LUSC and their effects on patient survival rates with a statistically significant difference of $\mathrm{P}<0.01$. The interaction of E2F3 with other proteins was analyzed using GeneMANIA (http://genemania.org/) (17).

The biological processes and pathway analyses of 15 E2F3 and E2F3-associated genes, based on the protein-protein interaction data were performed using the Protein Analysis THrough Evolutionary Relationships (PANTHER) online classification tool with a cut-off of $\mathrm{P}<0.05$ (http://www.pantherdb.org/).

Cell lines and cell culture. A human NSCLC A549 cell line was purchased from the Institute of Biochemistry and Cell Biology of the Chinese Academy of Sciences. The A549 cells were cultured in RPMI-1640 medium (Hyclone; GE Healthcare Life Sciences), supplemented with $10 \%$ fetal bovine serum (FBS, Gibco; Thermo Fisher Scientific Inc.), 2 mM L-glutamine (Gibco; Thermo Fisher Scientific Inc.), 100 units/ml penicillin and $100 \mu \mathrm{g} / \mathrm{ml}$ streptomycin (Gibco; Thermo Fisher Scientific Inc.). The cell line was cultured at $37^{\circ} \mathrm{C}$ in a humidified atmosphere with $5 \% \mathrm{CO}_{2}$.

Cell transfection. The cells were transfected with $50 \mathrm{nM}$ small interfering (si)RNAs targeting at E2F3 (targeting sequence \#1, GCGATCTCTTCGATGCTTA; targeting sequence \#2, AGA CCAAACTGTTATAGTT; targeting sequence \#3, CATTGA GGTTTACTTATGT; Guangzhou RiboBio Co., Ltd.) when the cell density reached $80-90 \%$ confluence. Lipofectamine ${ }^{\circledR} 2000$ reagent (Thermo Fisher Scientific, Inc.) was used for siRNA transfection at room temperature for $6 \mathrm{~h}$ before change of transfection medium to complete RPMI-1640 with $10 \%$ FBS. Non-specific siRNA (scrambled control)-transfected cells served as a negative control. The subsequential protein and functional analyses were performed 24,48 and $72 \mathrm{~h}$ after transfection.

Cell Counting Kit-8 (CCK-8) assay. The cells were seeded in 96 -well plates $\left(5 \times 10^{3}\right.$ cells/per well) for $24 \mathrm{~h}$ before transfection. After transfection with siRNA, the cells were incubated at $37^{\circ} \mathrm{C}$ in a humidified atmosphere with $5 \% \mathrm{CO}_{2}$. Next, $10 \mu \mathrm{l}$ CCK-8 solution (MedChemExpress LLC) was added to each well at 24, 48 and $72 \mathrm{~h}$. After incubation for $1 \mathrm{~h}$, absorbance was measured at $450 \mathrm{~nm}$ using a microplate reader.

Invasion and motility assays. For the invasion assay, after transfection for $24 \mathrm{~h}$, the cells were resuspended in serum-free RPMI-1640 medium in the upper chamber at a cell density of 100,000 cells/well of the Matrigel-coated chamber, which was pre-coated at $37^{\circ} \mathrm{C}$ for $2 \mathrm{~h}$ before cell seeding. Complete RPMI-1640 medium with 10\% FCS was added to the lower chamber. After wiping off the upper chamber cells with a cotton swab, the cells were incubated for $48 \mathrm{~h}$, followed by fixation with $4 \%$ paraformaldehyde at room temperature for $10 \mathrm{~min}$ and staining with $0.2 \%$ crystal violet at room temperature for $30 \mathrm{~min}$. Invaded cells on the underlying layer of the chamber were counted in 6 different regions at x100 magnification under a light microscope. 
A

\begin{tabular}{|l|c|c|}
\hline Analysis Type by Cancer & \multicolumn{3}{|l|}{} \\
\hline Bladder Cancer & 1 & \\
\hline Brain and CNS Cancer & 1 & 1 \\
\hline Breast Cancer & 3 & \\
\hline Cervical Cancer & 3 & \\
\hline Colorectal Cancer & 2 & \\
\hline Esophageal Cancer & 5 & \\
\hline Gastric Cancer & 3 & \\
\hline Head and Neck Cancer & 9 & \\
\hline Kidney Cancer & 1 & \\
\hline Leukemia & & 2 \\
\hline Liver Cancer & 4 & \\
\hline Lung Cancer & 5 & \\
\hline Lymphoma & & 1 \\
\hline Melanoma & 2 & \\
\hline Myeloma & & \\
\hline Other Cancer & 7 & \\
\hline Ovarian Cancer & 3 & \\
\hline Pancreatic Cancer & 1 & \\
\hline Prostate Cancer & & \\
\hline Sarcoma & 4 & \\
\hline Significant Unique Analyses & 53 & 4 \\
\hline Total Unique Analyses & 438 \\
\hline
\end{tabular}

$15^{10}{ }^{10} 5^{5} 1$

Median gene rank for all analyses
B

Comparison of E2F3 Across 19 Analyses

Over-expression / Copy Number Gain

Median rank:955.0 $P$ value:0.010 Gene:E2F3

\begin{tabular}{|l|l|l|l|l|l|l|l|l|l|l|l|l|l|l|l|l|l|l|}
\hline & & & & & & & & & & & & & & \\
\hline 1 & 2 & 3 & 4 & 5 & 6 & 7 & 8 & 9 & 10 & 11 & 12 & 13 & 14 & 15 & 16 & 17 & 18 & 19 \\
\hline
\end{tabular}

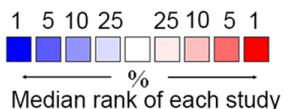

C

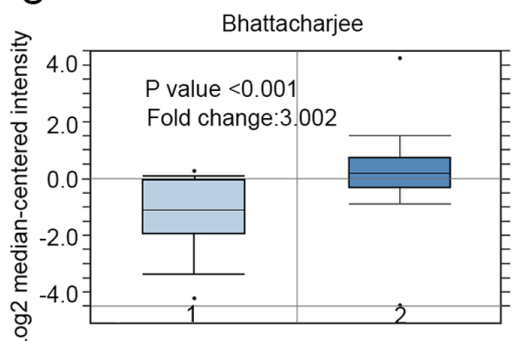

1. Lung (17) 2. Lung adenocarcinoma (132)

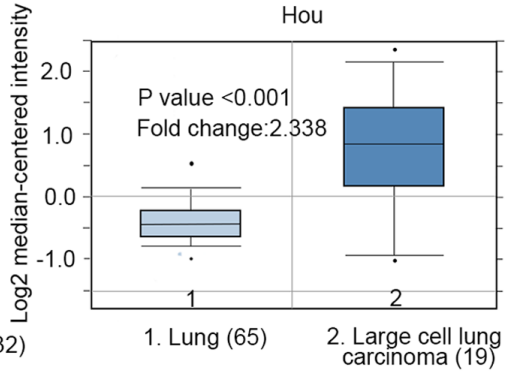

Su
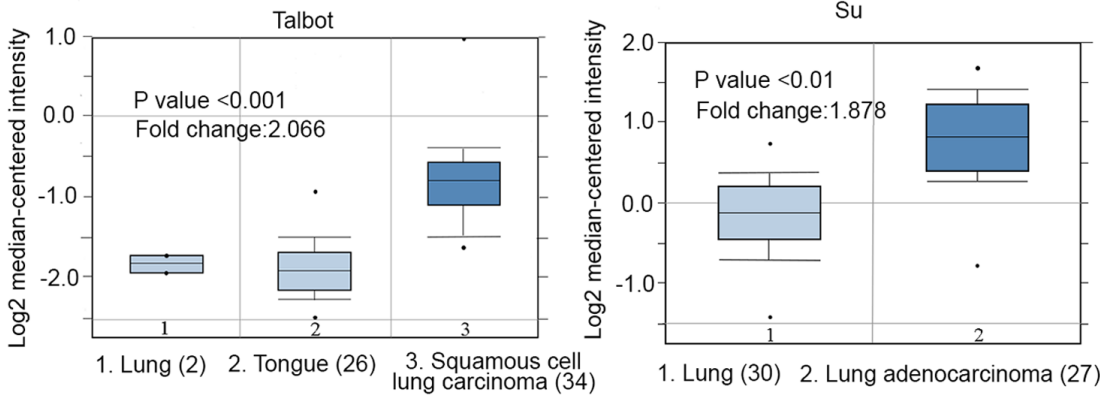

D

E2F3 differential plot

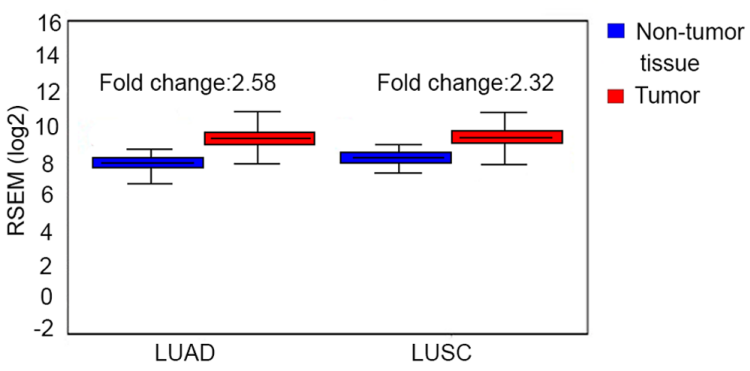

Figure 1. Expression level of E2F3 mRNA in patients with lung cancer from different databases. (A) Expression level of E2F3 from the Oncomine database. Red and blue indicates high and low expression, respectively in the different types of cancer compared with that in samples from non-tumor tissues. The darker the color, the more notable the difference in the ranking. The ranking of the relevant studies was performed according to the median value of E2F3 in the best study in each group. (B) Expression level of E2F3 mRNA in patients with lung cancer from the Oncomine database. The ranking was determined using the median value of E2F3 across each of the analyses. (C) E2F3 mRNA expression level in lung cancer tissues was higher compared with that in normal tissues in four cohorts. For Bhattacharjee, Hou and Su cohorts, lung tumor tissues were compared with non-tumor lung tissues. For Talbot cohort, lung tumor tissues were compared with non-tumor lung tissues and squamous cell epithelium from the tongue. Tumor entities compared in each cohort are indicated in the figure. (D) E2F3 was overexpressed in patients with LUAD or LUSC according to The Cancer Genome Atlas database. LUAD, lung adenocarcinoma; LUSC, lung squamous cell carcinoma; RSEM, RNA-sequencing by Expectation Maximization. Non-tumor tissue lung tissue from patients without malignancies.

A wound healing assay was then used to evaluate the motility of the A549 cells. The cells were cultured in RPMI-1640 medium with 10\% FBS for the sustenance of cell viability according to a protocol previously discussed (18). A wound was created by scraping the cell monolayer at $100 \%$ confluency with a $200 \mu \mathrm{l}$ pipette tip. The cells on the culture dish were incubated at $37^{\circ} \mathrm{C}$ for $72 \mathrm{~h}$. Images were captured at 0,24 and $48 \mathrm{~h}$ using a light microscope (magnification, $\mathrm{x} 100$ ). Image J v.2 software (National Institutes of Health) was used to calculate the wound healing rate (19).
Statistical analysis. Statistical analysis was performed either using SPSS v16.0 (SPSS, Inc.) and GraphPad Prism v8 (GraphPad Software, Inc.) software. The association between E2F3 expression level and the clinicopathological features of the patients with NSCLC was analyzed by grouping patients according to E2F3 IHC scores (high-expression, 3-12; low-expression, 0 -2). Statistical analysis of clinicopathological features was performed by using a $\chi^{2}$ test. For an expected count $<5$, the Fisher's exact test was used. Comparisons of mRNA and protein expression levels between two groups were 
Table II. miRNAs targeting E2F3 were downregulated in the subgroups of lung cancer patients with LUAD or LUSC.

A, miRNAs in LUSC

\begin{tabular}{|c|c|c|c|c|c|}
\hline miRNA & P-value & T-test FDR & $\begin{array}{c}\text { Tissue } \\
\text { upregulated in }\end{array}$ & $\begin{array}{l}\log _{2} \text { expression } \\
\text { level in tumor }\end{array}$ & $\begin{array}{c}\log _{2} \text { expression level in } \\
\text { normal tissue }\end{array}$ \\
\hline hsa-miR-101-3p & $6.49 \times 10^{-16}$ & $4.60 \times 10^{-14}$ & Normal & 13.56 & 15.14 \\
\hline hsa-miR-125b-5p & $4.74 \times 10^{-3}$ & $1.15 \times 10^{-2}$ & Normal & 9.25 & 9.63 \\
\hline hsa-miR-128-3p & $9.52 \times 10^{-12}$ & $1.14 \times 10^{-10}$ & Tumor & 8.05 & 6.82 \\
\hline hsa-miR-141-3p & $7.70 \times 10^{-12}$ & $9.52 \times 10^{-11}$ & Tumor & 9.66 & 8.12 \\
\hline hsa-miR-195-3p & $2.05 \times 10^{-9}$ & $1.53 \times 10^{-8}$ & Normal & 1.65 & 3.04 \\
\hline hsa-miR-210-3p & $1.30 \times 10^{-20}$ & $5.07 \times 10^{-18}$ & Tumor & 11.08 & 6.16 \\
\hline hsa-miR-221-3p & $2.16 \times 10^{-2}$ & $4.40 \times 10^{-2}$ & Normal & 8.33 & 8.66 \\
\hline hsa-miR-32-5p & $1.11 \times 10-^{3}$ & $2.99 \times 10^{-3}$ & Normal & 4.53 & 4.99 \\
\hline hsa-miR-34a-5p & $1.12 \times 10^{-4}$ & $3.62 \times 10^{-4}$ & Normal & 7.35 & 7.79 \\
\hline hsa-miR-34c-5p & $1.33 \times 10^{-4}$ & $4.23 \times 10^{-4}$ & Normal & 4.48 & 6.33 \\
\hline
\end{tabular}

B, miRNAs in LUAD

\begin{tabular}{|c|c|c|c|c|c|}
\hline miRNA & P-value & T-test FDR & $\begin{array}{l}\text { Tissue } \\
\text { upregulated in }\end{array}$ & $\begin{array}{l}\log _{2} \text { expression } \\
\text { level in tumor }\end{array}$ & $\begin{array}{c}\log _{2} \text { expression level in } \\
\text { normal tissue }\end{array}$ \\
\hline hsa-miR-125b-5p & $2.59 \times 10^{-2}$ & $6.16 \times 10^{-2}$ & Normal & 8.41 & 8.85 \\
\hline hsa-miR-128-3p & $4.2 \times 10^{-9}$ & $7.41 \times 10^{-8}$ & Tumor & 7.04 & 6.12 \\
\hline hsa-miR-141-3p & $1.69 \times 10^{-7}$ & $2.09 \times 10^{-6}$ & Tumor & 9.21 & 7.25 \\
\hline hsa-miR-195-3p & $4.24 \times 10^{-7}$ & $4.84 \times 10^{-6}$ & Normal & 1.19 & 2.26 \\
\hline hsa-miR-210-3p & $8.74 \times 10^{-21}$ & $3.40 \times 10^{-18}$ & Tumor & 8.34 & 4.02 \\
\hline hsa-miR-32-5p & $2.89 \times 10^{-2}$ & $6.62 \times 10^{-2}$ & Normal & 4.36 & 4.96 \\
\hline hsa-miR-34a-5p & $1.76 \times 10^{-6}$ & $1.73 \times 10^{-5}$ & Normal & 6.42 & 7.29 \\
\hline
\end{tabular}

FDR, false discovery rate; miR, microRNA; LUAD, lung adenocarcinoma; LUSC, lung squamous cell carcinoma.

performed either using a Student's t-test and the non-parametric Mann-Whitney U test. One-way ANOVA, followed by Tukey's HSD post hoc test, was used for the comparisons of datasets containing multiple groups. Wilcoxon test was used to analyze the IHC scores of paired tissue samples. Spearman's correlation was performed for the co-expression of the proteins of interest in the TMA. $\mathrm{P}<0.05$ (two-sided) was considered to indicate a statistically significant difference.

\section{Results}

Bioinformatics analysis reveals a high expression level of E2F3 in NSCLC. To verify the association between E2F3 expression level and lung cancer progression, data were downloaded from the Oncomine database. A total of 438 results associated with the mRNA expression level of E2F3 in different tumor types were found. Among these data, 58 studies showed significant differences between tumors and surrounding non-tumor tissues $(\mathrm{P}<0.01)$. Specifically, 54 studies reported an upregulation of E2F3, and 4 studies reported a downregulation of E2F3 in tumor tissues, as compared with that in the non-tumor tissues (Fig. 1A). Among the different tumor types, E2F3 expression level was highest in cervical cancer (3 studies), esophageal cancer (5 studies), lung cancer ( 5 studies), melanoma ( 2 studies) and ovarian cancer (3 studies), according to the ranking of the studies. Considering there are 5 studies supporting the upregulation of E2F3 in lung cancer, it was selected for further investigation in the present study. The expression level of E2F3 was further investigated in 19 lung cancer studies, which revealed an upregulation of E2F3 in tumor tissue, as compared with that in unpaired non-tumor tissues (fold change, >1.0). By comparing the E2F3 mRNA expression levels in each of the studies, 4 studies were selected due to the significant differences $(\mathrm{P}<0.01)$ identified between the tumor and non-tumor tissues (Fig. 1B). The studies included the Bhattacharjee, Hou, Talbot and Su cohorts (Fig. 1C). In the Bhattacharjee cohort, E2F3 was overexpressed in LUAD $(n=132)$, as compared with that in the non-tumor lung tissues without malignancy $(n=17$; fold change, 3.0). In the Hou cohort, E2F3 expression level was compared between large cell lung carcinoma $(n=19)$ and non-tumor lung tissues $(n=65$; fold change, 2.3). In the Talbot cohort, E2F3 was overexpressed in squamous cell carcinoma $(n=34)$, as compared with squamous cell epithelium from tongue tissue $(n=26$; fold change, 1.7). In the Su cohort, E2F3 was elevated in adenocarcinoma $(n=27)$, as compared with that in the non-tumor lung tissues without malignancy $(n=30$; fold change, 1.9). The results 
A

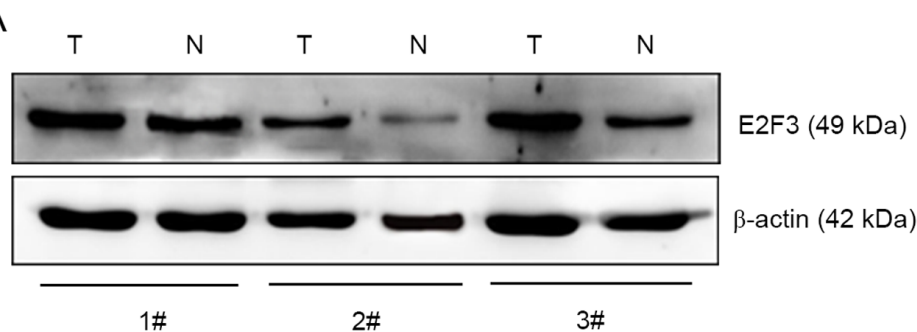

B
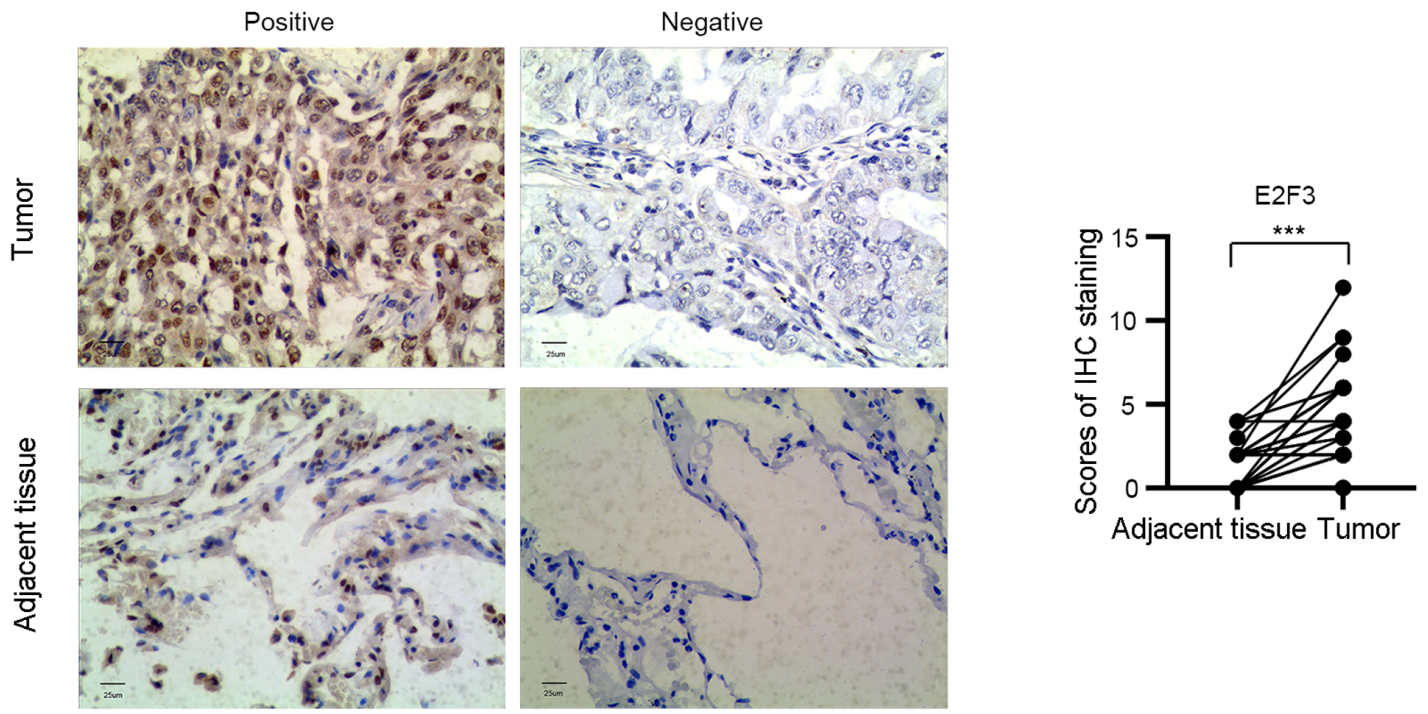

C

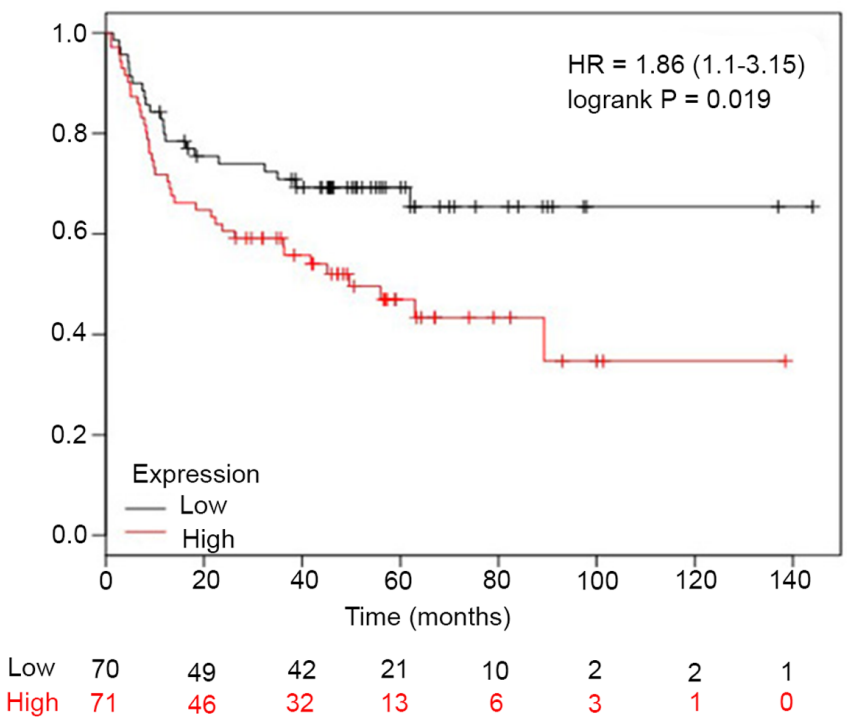

Figure 2. E2F3 protein expression level in lung cancer and corresponding normal tissues in the tissue microarray. (A) Western blot analysis of E2F3 protein expression level in lung cancer tissues $(n=3)$ and paired adjacent normal tissues $(n=3)$. $\beta$-actin served as a loading control. (B) Representive IHC staining of E2F3 in lung cancer and corresponding normal tissue (x400). The expression level of E2F3 in lung cancer was significantly higher compared with that in normal tissues $(\mathrm{n}=50)$. (C) The Kaplan-Meier survival curves showed that patients with E2F3 overexpression had a shorter survival time ${ }^{* * * *} \mathrm{P}<0.01$. IHC, immunohistochemistry; HR, hazard ratio.

were confirmed using TCGA database, where E2F3 mRNA was significantly overexpressed in LUAD tissue $(n=517)$, as compared with that in non-tumor tissues ( $\mathrm{n}=59$; fold change, 2.6; $\mathrm{P}<0.01)$. E2F3 mRNA was also overexpressed in squamous cell carcinoma tissue $(\mathrm{n}=501)$, as compared with that in non-tumor tissues without malignancy ( $\mathrm{n}=51$; fold change, 2.3; $\mathrm{P}<0.01$; Fig. 1D). These results from the mRNA-based databases revealed an association between E2F3 expression level and NSCLC tumorigenesis.

Overexpression of E2F3 in NSCLC tissues is associated with patient survival. To investigate the expression level of the $\mathrm{E} 2 \mathrm{~F} 3$ protein in tumors, the $\mathrm{E} 2 \mathrm{~F} 3$ protein expression level was analyzed in fresh tumor $(n=3)$ and respective surrounding 
A

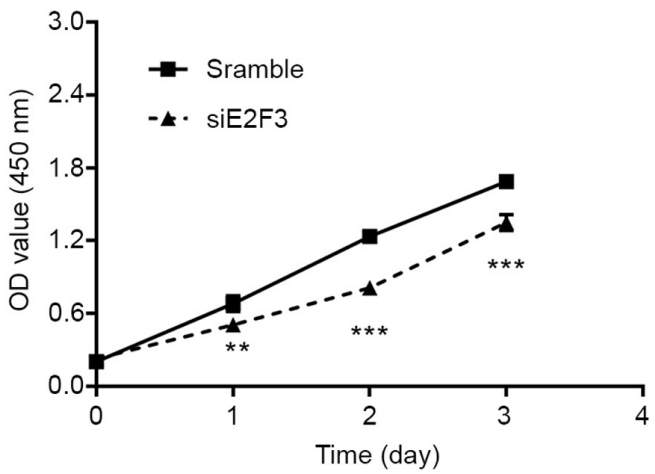

B
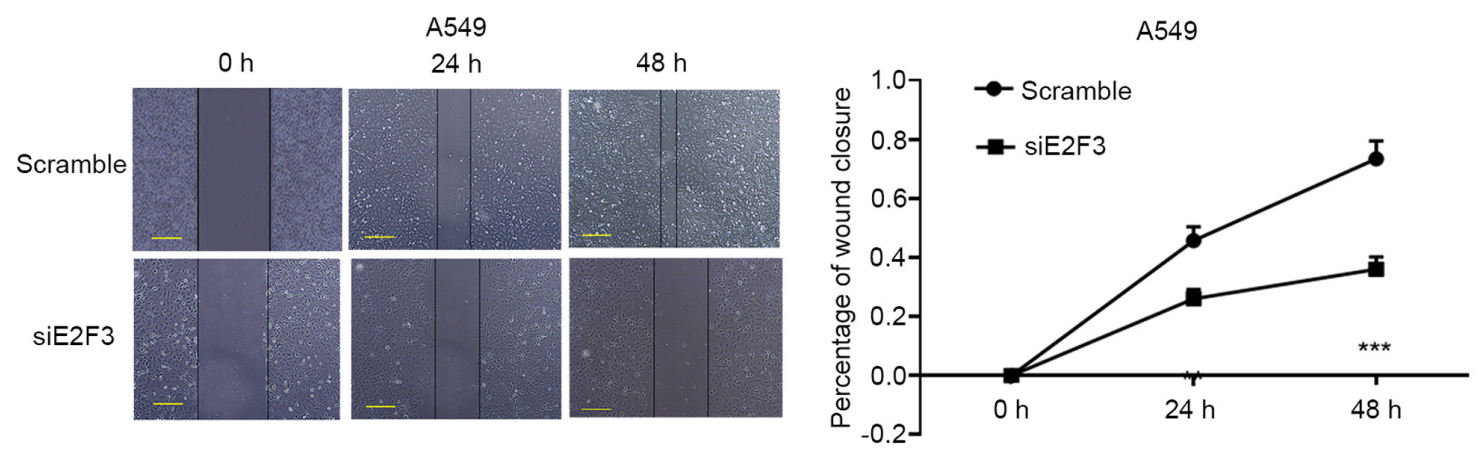

C
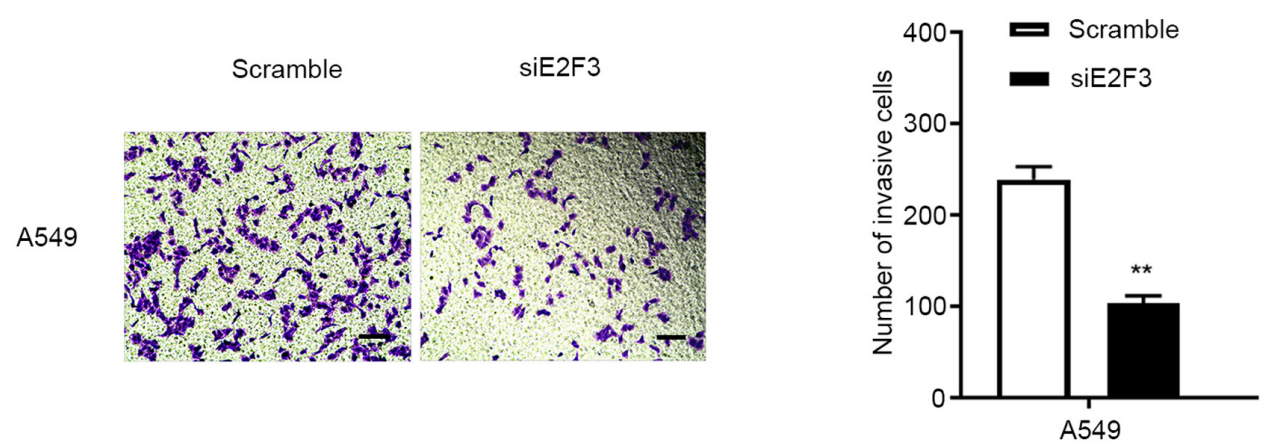

Figure 3. Inhibition of E2F3 expression level reduces cell proliferation and suppresses the migration and invasion of the A549 cells. (A) Cell proliferation was detected using a Cell Counting Kit-8 assay. The cells were transfected with siRNA against E2F3 or non-specific siRNA (scrambled control). (B) Wound-healing assay in the A549 cells transfected with siRNA against E2F3 or non-specific siRNA (scrambled control). The data was analyzed using a Student's t-test. (C) Matrigel assay was used to detect the effect of E2F3 siRNA on the invasion of the A549 cell line. The cells were transfected with siRNA against E2F3 or non-specific siRNA (scrambled control). Scale bar, $100 \mu \mathrm{m}$. The data was analyzed using a Student's t-test. ${ }^{* *} \mathrm{P}<0.01,{ }^{* * *} \mathrm{P}<0.001$. si, small interfering; OD, optical density.

adjacent lung tissue $(\mathrm{n}=3)$ from obtained from 50 patients with NSCLC. Western blot analysis showed that E2F3 protein expression level was markedly upregulated in fresh lung cancer tissues, as compared with non-tumor tissues $(\mathrm{P}<0.05$; Fig. 2A). To further investigate the expression level of E2F3 in NSCLC tissues, a TMA containing NSCLC tumor $(n=50)$ and the corresponding adjacent lung tissues $(\mathrm{n}=50)$ was prepared (Fig. S1). The IHC score of the E2F3 protein in the tumor tissues was significantly higher compared with that in the adjacent normal tissues (difference of median, 2.0; $\mathrm{P}<0.001$; Fig. 2B).

To determine the clinical relevance of E2F3 overexpression, survival analysis was performed. Kaplan-Meier Plotter was used and the results revealed that the patients in the high E2F3 mRNA expression subgroup had a worse OS time, as compared with that in patients in the low expression subgroup within 2.5 years $(\mathrm{P}=0.019$; Fig. $2 \mathrm{C})$. Furthermore, the clinical parameters of the 50 patients with NSCLC were collected and compared in the E2F3 high-expression (IHC score, 3-12; $n=29$ ) and low-expression (IHC score, $0-2 ; n=21$; Table I) groups. The statistical results showed that E2F3 overexpression had no association with sex, age, tumor diameter or TNM stage $(\mathrm{P}<0.05)$. However, E2F3 overexpression was significantly associated with lymphatic metastasis $(75.9 \%$ in the high-expression group; $\mathrm{P}<0.01$ ).

Downregulation of E2F3 inhibits cell proliferation and promotes apoptosis in the NSCLC cell line. To investigate the role of E2F3 in lung cancer, the A549 NSCLC cell line was used for the in vitro studies. siRNAs targeting 

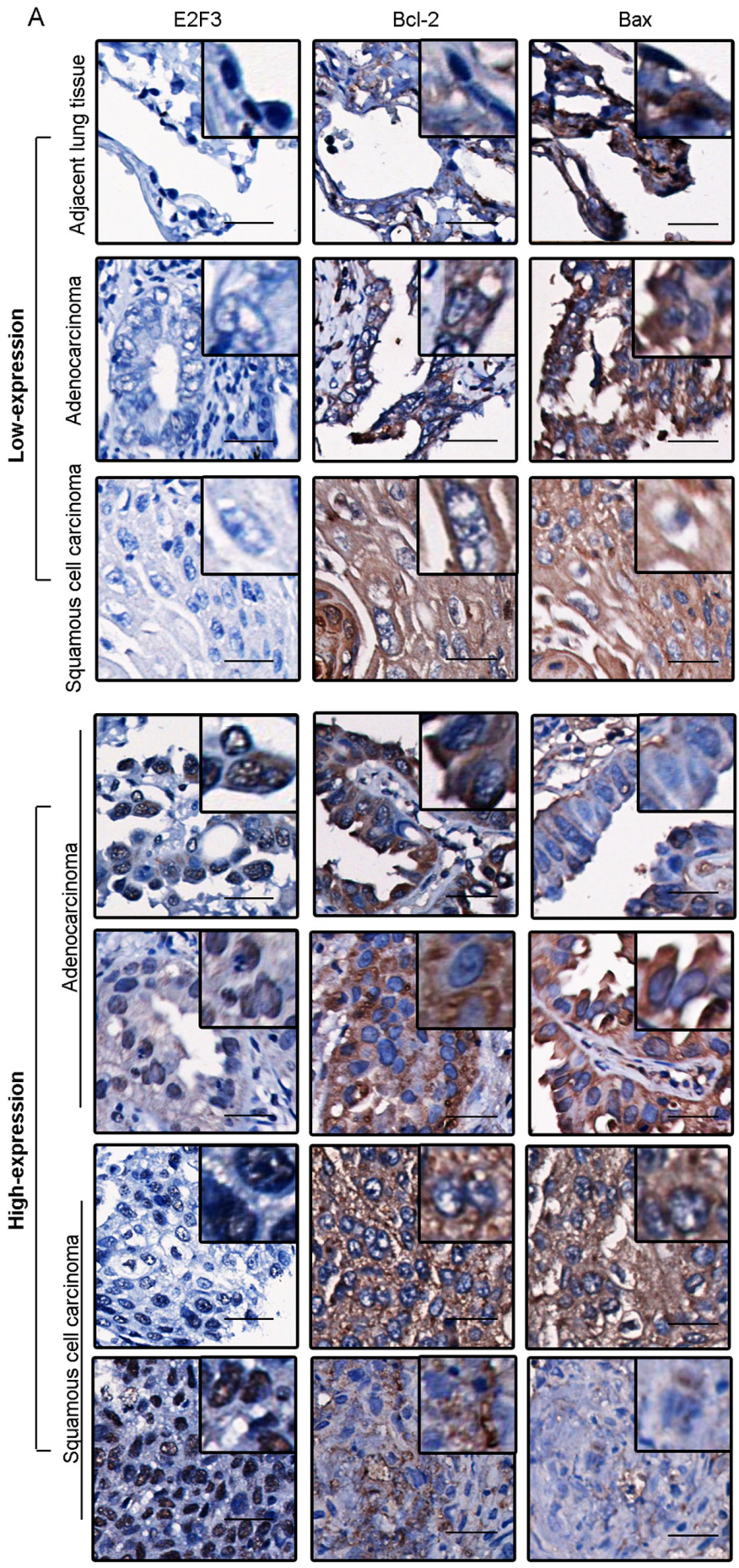

B
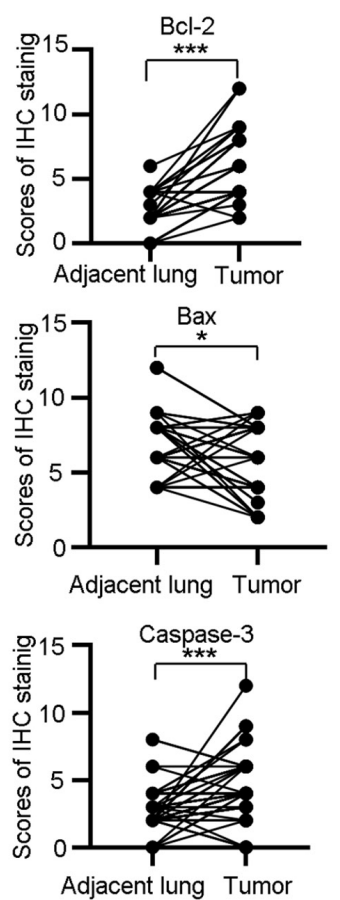

Figure 4. Immunohistochemistry analysis of E2F3, caspase-3, Bax and Bcl-2 in the tissue microarray. (A) Immunohistochemical staining of proteins in adjacent lung tissue, lung adenocarcinoma and squamous cell carcinoma. Scale bar, $200 \mu \mathrm{m}$. (B) Graph of immunohistochemical staining scores. Wilcoxon test was used to analyze the data. ${ }^{*} \mathrm{P}<0.05,{ }^{* * *} \mathrm{P}<0.001$.

E2F3 mRNA were designed and transfected into NSCLC A549 cell line. As compared with the non-specific siRNA (scrambled control)-transfected cells, the third E2F3 siRNA with the highest silencing efficiency was used in subsequent experiments (Fig. S2). The CCK-8 assay revealed that E2F3 knockdown significantly suppressed NSCLC cell proliferation in the A549 cells (Fig. 3A).

To confirm the effect of E2F3 on apoptosis in tissues, IHC staining was performed on the TMA sections from 50 patients with NSCLC, to investigate the expression of Bcl-2, Bax and caspase-3 (Fig. 4A). The IHC scores at different stages of LUSC $(n=23)$ and LUAD $(n=27)$ were compared with their respective surrounding non-tumor lung tissues (Fig. 4B). Of note, Bcl-2 was overexpressed in the tumor tissues, as compared with that in the non-tumor tissues (median of differences, 4.0; $\mathrm{P}<0.001$ ). By contrast, Bax was downregulated in tumor tissues, as compared with that in the non-tumor tissues (median of differences $=-0.5$; $\mathrm{P}<0.05)$. Caspase-3 was also upregulated in the NSCLC tissue samples (median of differences, 2.0; $\mathrm{P}<0.001$ ). A correlation test was performed between the nuclear expression of E2F3 with 
A

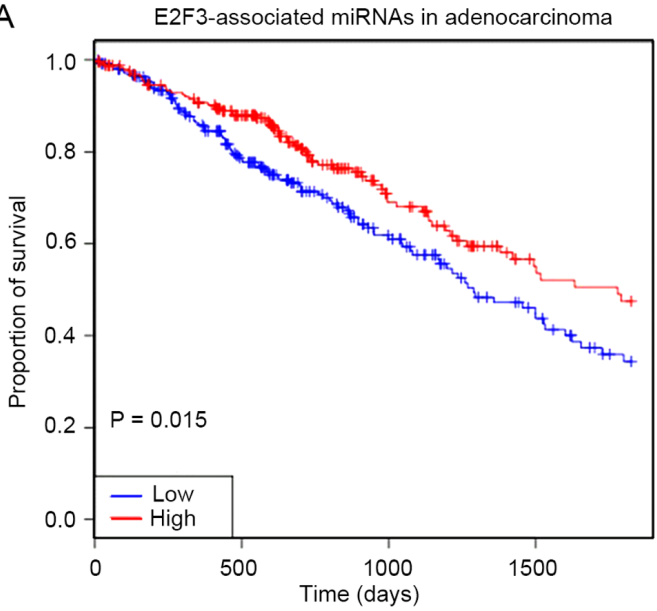

B

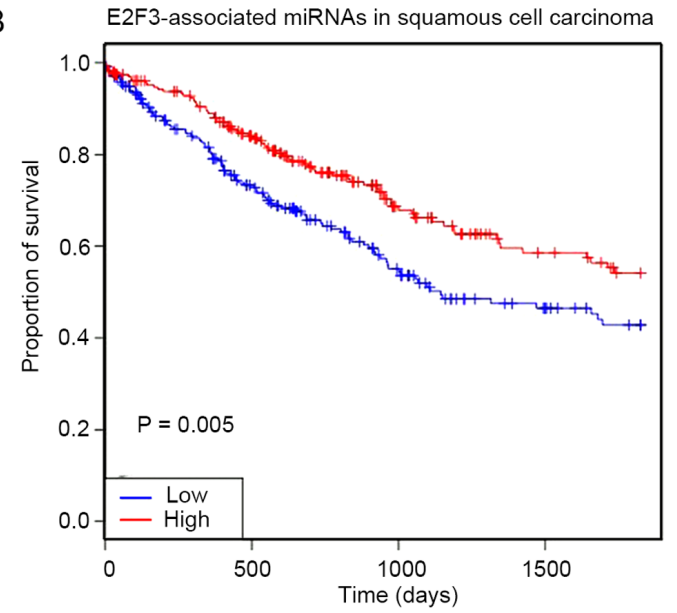

Figure 5. Survival analysis of E2F3-associated miRNAs in patients with non-small cell lung cancer. Survival curves showing the proportion of patients grouped by the expression levels of miRNAs associated with E2F3 in (A) lung adenocarcinoma and (B) squamous carcinoma. Log-rank test was used to statistically compare the groups. Statistical significance, $\mathrm{P}=0.015$ for adenocarcinoma and $\mathrm{P}=0.005$ for squamous carcinoma. miRNA, microRNA.

IHC scores of Bcl-2, Bax and Caspase-3 in tumor samples. Bcl-2 was positively correlated with E2F3 expression level $(r=0.32$; $\mathrm{P}=0.02$ ), while Bax expression level was negatively correlated with E2F3 expression level, although this was not statistically significant ( $\mathrm{r}=-0.22, \mathrm{P}=0.13$ ). Caspase- 3 did not show correlation with $\mathrm{E} 2 \mathrm{~F} 3$ expression in the patients analyzed $(\mathrm{r}=0.13 ; \mathrm{P}=0.37)$.

E2F3 promotes tumor cell migration and invasion in NSCLC cells. The association between E2F3 mRNA expression levels with lymphatic spreading in patients with NSCLC revealed a potential role of E2F3 in the regulation of tumor cell invasiveness. To validate the biological function of E2F3 in NSCLC, wound healing and Matrigel assays were performed in the A549 cell line. The knockdown of E2F3 expression by siRNA significantly inhibited tumor cell migration at 24 and $48 \mathrm{~h}$ in A549 cell (Fig. 3B). Furthermore, the Matrigel assay detected a reduction in invasive cells following the inhibition of E2F3 expression in A549 cell (fold change, 0.43; $\mathrm{P}<0.01$; Fig. 3C). These data revealed the potential role of E2F3 in the regulation of NSCLC invasiveness, in addition to its effect on uncontrolled tumor proliferation.

Downregulation of E2F3 upstream miRNAs is associated with poor prognosis in NSCLC. The top 10 confirmed miRNAs targeting E2F3 were investigated using Exiqon (https://www. exiqon.com/miRSearch). The OncomiR database showed that the expression levels of these 10 E2F3 miRNAs were significantly different between LUSC and normal tissues (Table II). Among the 10 miRNAs, 7 were overexpressed in normal tissues. In addition, 7 miRNAs exhibited a statistically significant difference in expression between LUAD and normal tissues, among which 3 exhibited an upregulation in tumor tissues compared with normal tissues. From the OncomiR database, survival analysis showed that the lower expression level of E2F3-associated miRNAs in LUSC and LUAD was correlated with poor prognosis in patients within 5 years (Fig. 5).

Protein-protein interaction network of E2F3 and biological process annotation. GeneMANIA was used to analyze the protein-protein interactions between E2F3 and other proteins. The results showed that E2F3 interacted directly with a total of 15 proteins (Fig. S3A) and among them, E2F3 had a physical interaction with TEA domain transcription factor-3, transcription factor binding to IGHM enhancer-3, lethal (3) malignant brain tumor like-2, vacuolar protein sorting-associated protein-72, RING1 and YY1 binding protein, transcription factor SP1 and bromodomain containing-8. E2F3 was co-expressed with E74 like ETS transcription factor-1, ribonucleotide reductase catalytic subunit M1 and thymidylate synthetase. E2F3 was also co-expressed and co-localized with DNA methyltransferase-1. E2F3 was co-localized and had strong physical interactions with cyclin A2 and MutS homolog-2, had physical interactions and similar protein domains with transcription factor Dp-1, and was co-expressed and shared the same protein domains with E2F2. Biological pathway enrichment and biological process annotation was performed on the genes that had protein-protein interactions with E2F3 using the PANTHER database. The pathway enrichment analysis revealed 8 biological processes $(\mathrm{P}<0.05$; Fig. S3B).

\section{Discussion}

Lung cancer is the leading cause of cancer-specific mortality in China, and NSCLC is the most common type of lung cancer tumor (1). Numerous advances have been made in the molecular diagnosis of lung cancer; however, the survival rate of patients with this malignant tumor remains low (20). Therefore, the identification of new biomarkers is important to improve the subclassification of NSCLC for the planning of appropriate treatment, and establishment of a prognostic evaluation system (21).

The E2F family includes important transcriptional factors responsible for the regulation of cell mitosis and control of cell cycle (11-15). The overexpression of E2F was reported in human gastric cancer, colon cancer and hepatocellular carcinoma and was also found in neuroendocrine tumors of the lung (22-24). In gastric cancer, the non-coding small 
RNA miR-449a could affect the proliferation and apoptosis of cancer cells by inhibiting the expression level of E2F3 (25). These studies illustrated multiple biological functions of the E2F family in regulating the development of different tumors.

Bioinformatics has become an important tool for biomedical research. Databases from large-scale study samples have provided convincing data with reduced errors (26). The Oncomine and TCGA databases were used to examine the expression level of E2F3 mRNA in lung cancer and non-tumor tissue from patients without malignancies. The results showed that the mRNA expression level of E2F3 in lung cancer tissues was significantly higher compared with that in normal tissues, suggesting that E2F3 might play a vital role in the development of lung cancer. Due to the diversity of lung cancer subtypes, the expression level of E2F3 mRNA was analyzed in different histological subtypes. E2F3 mRNA expression level was significantly overexpressed in adenocarcinoma and squamous cell carcinoma, which suggested a wide effect of E2F3 in the development of NSCLC.

To investigate whether a miRNA-mRNA interaction was involved in the development of NSCLC, the Exiqon database was used to identify the top 10 confirmed miRNAs targeting E2F3. The results of the OncomiR database analysis showed that most of these 10 miRNAs had a low expression level in lung cancer, and survival analysis showed that the downregulation of the E2F3-associated miRNAs was associated with low survival rate in patients with lung cancer. This was consistent with the results that the overexpression of E2F3 mRNA was associated with low survival time in patients with LUSC and LUAD, suggesting that these miRNAs may play an important role in the progression of lung cancer through E2F3.

To evaluate the biological function of E2F3 in NSCLC development, siRNA-based inhibition of E2F3 was performed in a A549 lung cancer cell line. Functional analysis also supported the important function of E2F3 in promoting tumor cell proliferation. Other studies have reported the effect of E2F on tumor cell apoptosis in gastric cancer and head and neck carcinoma, suggesting an intensive impact of E2F3 in inhibiting tumor cell apoptosis $(25,27)$. From the IHC analysis of a NSCLC TMA, the overexpression of E2F3 was significantly correlated with the expression level of Bcl-2 in adenocarcinoma and squamous cell carcinoma tissues (Fig. 4A). E2F3 expression level was negatively correlated with the pro-apoptotic regulator Bax; however, the result was not statistically significant. These data in the lung cancer cell line and the TMA suggested that the anti-apoptotic effect of E2F3 was exerted through its regulation of Bcl-2 expression.

Clinical data from the same cohort of patients with NSCLC $(n=50)$ showed no association between the high expression level of E2F3, and sex, age, tumor diameter, differentiation degree and TNM stage $(\mathrm{P}>0.05)$; however an association was found with lymph node metastasis $(\mathrm{P}<0.05)$. E2F3 was widely overexpressed in patients with lymph node metastasis $(75.9 \%$ of patients had a high E2F3 expression), as compared with that in patients without lymph node metastasis $(33.3 \%$ of patients had a high E2F3 high expression). In addition, studies have shown that miR-141 inhibited the proliferation and metastasis of gastrointestinal cancers by targeting E2F3 $(28,29)$. In the present study, E2F3 inhibition led to a reduction in tumor cell migration and invasion of the A549 cell line. In combination, these results revealed a potential role of E2F3 in regulating NSCLC invasiveness.

In the present study, the overexpression of E2F3 in lung cancer was verified by bioinformatics and clinical samples, which was associated with the progression of NSCLC, particularly in squamous cell lung carcinoma and adenocarcinoma. In A549 cell and human tissues, various biological functions of E2F3 in NSCLC have been identified, particularly through the inhibition of tumor cell apoptosis, which was associated with the overexpression of the anti-apoptotic regulator Bcl-2. As a result, the present study has revealed a new aspect, that E2F3 promoted tumor proliferation by inhibiting tumor cell apoptosis through the regulation of the Bcl-2 and apoptotic signaling pathway. The increased expression level of E2F3 in tumor tissue suggests that it could become a potential biomarker for the subclassification and the prediction in prognosis of NSCLC, which could also be used for the development of targeted therapy.

The important role of E2F3 in promoting tumor proliferation in the progression of NSCLC was identified in the present study. In the clinic, EGFR molecular assessment was routinely performed in patients with NSCLC for administration of TKIs, such as gefitinib and erlotinib (3). However, during the treatment of TKIs in patients with activating mutations in EGFR, tumor cells developed resistance to TKIs, where alterations of E2F-target genes were frequently found (30). In this situation, activation of E2F3 and related signaling pathway may account for the development of TKI-resistant population of tumor cells, which indicated the importance of detecting E2F3 expression level in parallel to EGFR for the treatment of patients with NSCLC.

Due to the limited number of fresh samples collected in the present study, expression level of E2F3 was only performed at the protein level and in paraffin-embedded tissue. The datasets of lung cancer cohorts (the Bhattacharjee, Hou, Talbot, and $\mathrm{Su}$ cohorts) were used for the selection of genes at the mRNA level. Transcriptional analysis of E2F3 will be performed in the future for the confirmation of the prognostic value of E2F3 in NSCLC.

\section{Acknowledgements}

Tissue samples were provided by the tissue bank of the Second Affiliated Hospital of Soochow University (Jiangsu, China) with the approval of the Ethics Committee of the Soochow University. The authors would like to thank Ms. Fei Zhao from the Sanitation and Environment Technology Institute of Soochow University (Jiangsu, China) for his technical support of tissue sample preparation and Ms. Yue Wu from the Sanitation and Environment Technology Institute of Soochow University (Jiangsu, China) for the performance of IHC staining.

\section{Funding}

The study was supported by the National Key Research and Development Program of China (grant no. 2016YFC1303800), the Priority Academic Program Development of Jiangsu Higher Education Institutions, the Natural Science Foundation of the Jiangsu Higher Education Institutions of China (grant no. 18KJB320017) and the Youth Program of National Natural Science Foundation of China (grant no. 81902400). 


\section{Availability of data and materials}

All data generated or analyzed during this study are included in this published article.

\section{Authors' contributions}

LW and SW performed the experiments, analyzed and interpreted the data, and wrote the manuscript. JL performed histological assessment of TMA from patients with NSCLC. YX performed the cell culture experiments and analyzed the data. XL analyzed the immunohistology data. SLW and MS designed the study. LW, SW and SLW confirm the authenticity of all the raw data. All authors have read and approved the manuscript.

\section{Ethical approval and consent to participate}

Tissue samples and clinical data collection were approved by the Ethics Committee of the Soochow University (Jiangsu, China). Written informed consent for publication was obtained from all participants.

\section{Patient consent for publication}

Not applicable.

\section{Competing interests}

The authors declare that they have no competing interests.

\section{References}

1. Chen WQ, Li H, Sun KX, Zheng RS, Zhang SW, Zeng HM, Zou XN, Gu XY and He J: Report of cancer incidence and mortality in China, 2014. Zhonghua Zhong Liu Za Zhi 40: 5-13, 2018 (In Chinese).

2. Oliver TG, Patel J and Akerley W: Squamous non-small cell lung cancer as a distinct clinical entity. Am J Clin Oncol 38: 220-226, 2015.

3. Osmani L, Askin F, Gabrielson E and Li QK: Current WHO guidelines and the critical role of immunohistochemical markers in the subclassification of non-small cell lung carcinoma (NSCLC): Moving from targeted therapy to immunotherapy. Semin Cancer Biol 52: 103-109, 2018.

4. Cancer Genome Atlas Research Network: Comprehensive molecular profiling of lung adenocarcinoma. Nature 511: 543-550, 2014.

5. Segundo-Val IS and Sanz-Lozano CS: Introduction to the gene expression analysis. Methods Mol Biol 1434: 29-43, 2016.

6. Rhodes DR, Yu J, Shanker K, Deshpande N, Varambally R, Ghosh D, Barrette T, Pandey A and Chinnaiyan AM: ONCOMINE: A cancer microarray database and integrated data-mining platform. Neoplasia 6: 1-6, 2004.

7. Gyorffy B, Surowiak P, Budczies J and Lánczky A: Online survival analysis software to assess the prognostic value of biomarkers using transcriptomic data in non-small-cell lung cancer. PLoS One 8: e82241, 2013.

8. Lánczky A, Nagy A, Bottai G, Munkácsy G, Szabó A, Santarpia L and Győrffy B: MiRpower: A web-tool to validate survival-associated miRNAs utilizing expression data from 2178 breast cancer patients. Breast Cancer Res Treat 160: 439-446, 2016.

9. Zhu Y, Peng Q, Lin Y, Zou L, Shen P, Chen F, Min M, Shen L, Chen J and Shen B: Identification of biomarker microRNAs for predicting the response of colorectal cancer to neoadjuvant chemoradiotherapy based on microRNA regulatory network. Oncotarget 8: 2233-2248, 2017.

10. Sarver AL, Sarver AE, Yuan C and Subramanian S: OMCD: OncomiR cancer database. BMC Cancer 18: 1223, 2018.
11. Zhan L, Zhang Y, Wang W, Song E, Fan Y and Wei B: E2F1: A promising regulator in ovarian carcinoma. Tumour Biol 37: 2823-2831, 2016.

12. Park SA, Platt J, Lee JW, López-Giráldez F, Herbst RS and Koo JS: E2F8 as a novel therapeutic target for lung cancer. J Natl Cancer Inst 107: 151, 2015.

13. Kurtyka CA, Chen L and Cress WD: E2F inhibition synergizes with paclitaxel in lung cancer cell lines. PLoS One 9: e96357, 2014.

14. Rotgers E, Rivero-Müller A, Nurmio M, Parvinen M, Guillou F, Huhtaniemi I, Kotaja N, Bourguiba-Hachemi S and Toppari J: Retinoblastoma protein (RB) interacts with E2F3 to control terminal differentiation of sertoli cells. Cell Death Dis 5: e1274, 2014.

15. Danielian PS, Friesenhahn LB, Faust AM, West JC, Caron AM, Bronson RT and Lees JA: E2f3a and E2f3b make overlapping but different contributions to total E2f3 activity. Oncogene 27: 6561-6570, 2008.

16. Lou X, Fu J, Zhao X, Zhuansun X, Rong C, Sun M, Niu H, Wu L, Zhang Y, An L, et al: MiR-7e-5p downregulation promotes transformation of low-grade follicular lymphoma to aggressive lymphoma by modulating an immunosuppressive stroma through the upregulation of FasL in M1 macrophages. J Exp Clin Cancer Res 39: 237, 2020.

17. Montojo J, Zuberi K, Rodriguez H, Bader GD and Morris Q: GeneMANIA: Fast gene network construction and function prediction for cytoscape. F1000Res 3: 153, 2014.

18. Zhang C, Liu J, Jin N, Zhang G, Xi Y and Liu H: SiRNA targeting mTOR effectively prevents the proliferation and migration of human lens epithelial cells. PLoS One 11: e0167349, 2016.

19. Collins TJ: ImageJ for microscopy. Biotechniques 43: 25-30, 2007.

20. Meyers DE, Bryan PM, Banerji S and Morris DG: Targeting the PD-1/PD-L1 axis for the treatment of non-small-cell lung cancer. Curr Oncol 25: e324-e334, 2018.

21. Guinde J, Frankel D, Perrin S, Delecourt V, Lévy N, Barlesi F, Astoul P, Roll P and Kaspi E: Lamins in lung cancer: Biomarkers and key factors for disease progression through miR-9 regulation? Cells 7: 78, 2018.

22. Suzuki T, Yasui W, Yokozaki H, Naka K, Ishikawa T and Tahara E: Expression of the E2F family in human gastrointestinal carcinomas. Int J Cancer 81: 535-538, 1999.

23. Oliveira A, Beyer G, Chugh R, Skube SJ, Majumder K, Banerjee S, Sangwan V, Li L, Dawra R, Subramanian S, et al: Triptolide abrogates growth of colon cancer and induces cell cycle arrest by inhibiting transcriptional activation of E2F. Lab Invest 95: 648-659, 2015.

24. Salon C, Merdzhanova G, Brambilla C, Brambilla E, Gazzeri S and Eymin B: E2F-1, Skp2 and cyclin E oncoproteins are upregulated and directly correlated in high-grade neuroendocrine lung tumors. Oncogene 26: 6927-6936, 2007.

25. Li X, Li H, Zhang R, Liu J and Liu J: MicroRNA-449a inhibits proliferation and induces apoptosis by directly repressing E2F3 in gastric cancer. Cell Physiol Biochem 35: 2033-2042, 2015.

26. Sherlock G and Ball CA: Storage and retrieval of microarray data and open source microarray database software. Mol Biotechnol 30: 239-251, 2005.

27. Frank DK, Liu TJ, Frederick MJ and Clayman GL: Combination E2F-1 and p53 gene transfer does not enhance growth inhibition in human squamous cell carcinoma of the head and neck. Clin Cancer Res 4: 2265-2272, 1998.

28. Zhou X, Ji G, Ke X, Gu H, Jin W and Zhang G: MiR-141 inhibits gastric cancer proliferation by interacting with long noncoding RNA MEG3 and down-regulating E2F3 expression. Dig Dis Sci 60: 3271-3282, 2015.

29. Xue J, Niu YF, Huang J, Peng G, Wang LX, Yang YH and Li YQ: MiR-141 suppresses the growth and metastasis of HCC cells by targeting E2F3. Tumour Biol 35: 12103-12107, 2014.

30. Naeini MM, Tavassoli M and Ghaedi K: Systematic bioinformatic approaches reveal novel gene expression signatures associated with acquired resistance to EGFR targeted therapy in lung cancer. Gene 667: 62-69, 2018.

This work is licensed under a Creative Commons Attribution-NonCommercial-NoDerivatives 4.0 International (CC BY-NC-ND 4.0) License. 